\title{
Progressive late cardiac dysfunctions chemo- and radiotherapy related in a middle age woman with Hodgkin lymphoma - a case report
}

\author{
Maria Andrada JIGA ${ }^{1}$, Mariana Cornelia TILINCA ${ }^{2}$, Dragos Gabriel IANCU ${ }^{1}$, \\ Paula Anca SULEA ${ }^{3}$, Marian POP2,4 \\ ${ }^{1}$ Internal Medicine - Cardiology II Unit, Emergency County Hospital Targu Mures, Romania \\ ${ }^{2}$ Department ME2, Faculty of Medicine in English, "G.E. Palade" University of Medicine, Pharmacy, \\ Science and Technology of Targu Mures, Romania \\ ${ }^{3}$ Emergency County Hospital Targu Mures, Romania \\ ${ }^{4}$ Radiology and Medical Imaging Clinic, Emergency County Hospital Targu Mures, Romania
}

\footnotetext{
ABSTRACT

Background. Elaborated chemotherapy protocols used for a broad spectrum of solid and haematological malignancies have a significant cardiotoxicity potential. Different biologic mechanisms generated by Doxorubicin used in $A B V D$ protocol, induce irreversible myocardial alterations, like type I cancer therapeutics-related cardiac dysfunction. The present case-report highlights the importance of comprehensive diagnostic evaluation of a common mitral valve disease and emphasizes the importance of the accurate etiology in a case of late cardiomyopathy anthracycline + radiation related.

Case presentation. A 46-year-old Caucasian woman was admitted in sequence of 20 years prior anthracycline chemotherapy + radiotherapy protocols (five MOPP cycles, one cycle of $A B V D$, and three radiotherapy cycles of mantle Cobalt-60 therapy) for stage IIB Hodgkin lymphoma, medical investigations supporting a diagnosis of cardiotoxicity. The optimal pharmacological regimen for heart failure was prescribed with significant improvement of symptoms six months later. Consecutive four-year regular follow-up, complete clinical workup, lab tests and high-performance imaging studies endorsed a slow progression of cardiomyopathy with the appearance of a left bundle branch block and a balanced clinical status. Except cardiac and thyroid involvement, no relapse of Hodgkin lymphoma, musculoskeletal disease or other malignancies were identified 24 years after chemotherapy and radiation.

Pathogenesis of late-onset chronic development cardiotoxicity anthracycline-containing chemotherapy + mediastinal radiotherapy related remains unclear, resulting from multifactorial and complex mechanisms, and usually is considered irreversible. Notably, long-term survivors who experienced Hodgkin lymphoma in childhood or young age, appear to be susceptible and remain at increased risk of developing heart failure decades after.
} 
Conclusions. From the setting-up of targeted therapy for Hodgkin lymphoma, cardio-oncology knowledge should be joined, avoiding the late stages, when symptomatic heart failure occurs. For Hodgkin lymphoma treated patients, serial follow-up and individualized approach are required to reduce the continuously increased risk of future $C V$ events.

Keywords: adult Hodgkin lymphoma survivor, late cardiotoxicity, heart failure, mitral regurgitation, cardiac conduction disorder, treatment, long term follow-up, case report

\section{INTRODUCTION}

Different chemotherapy protocols used for a broad spectrum of solid and haematological malignancies have a significant cardiotoxicity potential $(1,2)$. The use of chemo- and radiotherapies may amplify the risk of cardiovascular (CV) events up to three times for cancer survivors in terms of medium and long term prognosis $(3,4)$.

Although long-term cardiotoxicity is a well-recognized issue, anthracyclines are widely used in the treatment protocols of patients with Hodgkin lymphoma $(\mathrm{HL})$ and contribute substantially to improving the prognosis of these patients. Doxorubicin, used in ABVD protocol, induce type I cancer therapeutics-related cardiac dysfunction (CTRCD) via reactive oxygen species, and other different biologic mechanisms; myocardial alterations are irreversible and dose-related, especially if the cumulative dose exceeds $400 \mathrm{mg} / \mathrm{sqm}$ $(5,6)$. Late cardiac complications (heart failure, arrhythmias, valvular involvement, chronic coronary syndromes, pericardial disease, cerebrovascular disease) can occur variable (years or decades) after completion of anthracycline administration, expressly in adult patients who experienced cancer treatment in childhood $(7,8)$.

CRTCD, defined as a decrease of more than $10 \%$ of the left ventricular ejection fraction (LVEF), compared with pre-chemotherapy values and drop to less than $53 \%$, is associated with the occurrence of heart failure (HF) in $5 \%$ to $26 \%$ of cases, and $48 \%$ for left ventricular dysfunction, depending on the population and follow-up duration (9-13).

Anthracycline-induced cardiomyopathy (AICM) display a broad spectrum range from the asymptomatic decline in systolic function quantified through measurement of left ventricular ejection fraction (LVEF) to the development of heart failure.

The risk of AICM should be hypothesized in the presence of associated conditions such as female sex, Afro-American descendants, older age (> 65 years), pediatric population, chronic kidney disease, systemic hypertension, genomic variants, but factors triggering individual susceptibility are less known $(9,10,12,13)$. Retrospective evaluation of long $\mathrm{HL}$ who encountered combined therapy (anthracyclines plus early radiotherapy protocols) in childhood or adulthood underlines an unquestionably increased risk of development of any cardiovascular disease, coronary artery disease (CAD), and heart failure, after a median interval of 20 years (14).

Comparing AICM with the other forms of cardiomyopathies, this is associated with a poor prognosis, with a 1-year death rate of $50 \%$ up to $60 \%$ in the first two years after diagnosis, typically result from congestive heart failure (15). In 2000, Felker et al. reported a poor survival prognosis 3.5 in AICM patients compared to those with idiopathic dilated cardiomyopathy (DCM) (16).

The objective of this paper is to emphasize the importance of an appropriate etiological diagnosis of DCM and heart failure management in a middle-aged woman exposed to previous chemotherapies and radiotherapy (RT) for Hodgkin lymphoma along with relevant data regarding combined long term effects of appropriate therapies for this haematological malignancy.

\section{CASE PRESENTATION}

A 46-year-old Caucasian woman with a history of Hodgkin's lymphoma was admitted in 2015 for fatigue, palpitations, dizziness, shortness of breath, dyspnoea.

In 1995, she was diagnosed with HL stage IIB (Cotswold Modification of Ann Arbor staging system). No suggestive medical records (negative personal and family history for CV disease, rheumatic fever, recurrent viral infections, hormonal, and neoplastic disorders) were documented before cancer therapy. Chemotherapy (five cycles of MOPP and one cycle of ABVD) and RT ( 3 cycles of mantle therapy Cobalt-60) were started at the age of 22 , attaining complete remission in November 1996.

Unfortunately, after more than two decades, there were no evidences regarding cumulative dose of chemotherapeutic agents used, or the total mediastinal radiation dose. DCM, mitral regurgitation and heart failure were diagnosed in ambulatory settings.

On admission, physical examination revealed orthopnoea, bilateral limbs oedema, bilateral pulmo- 


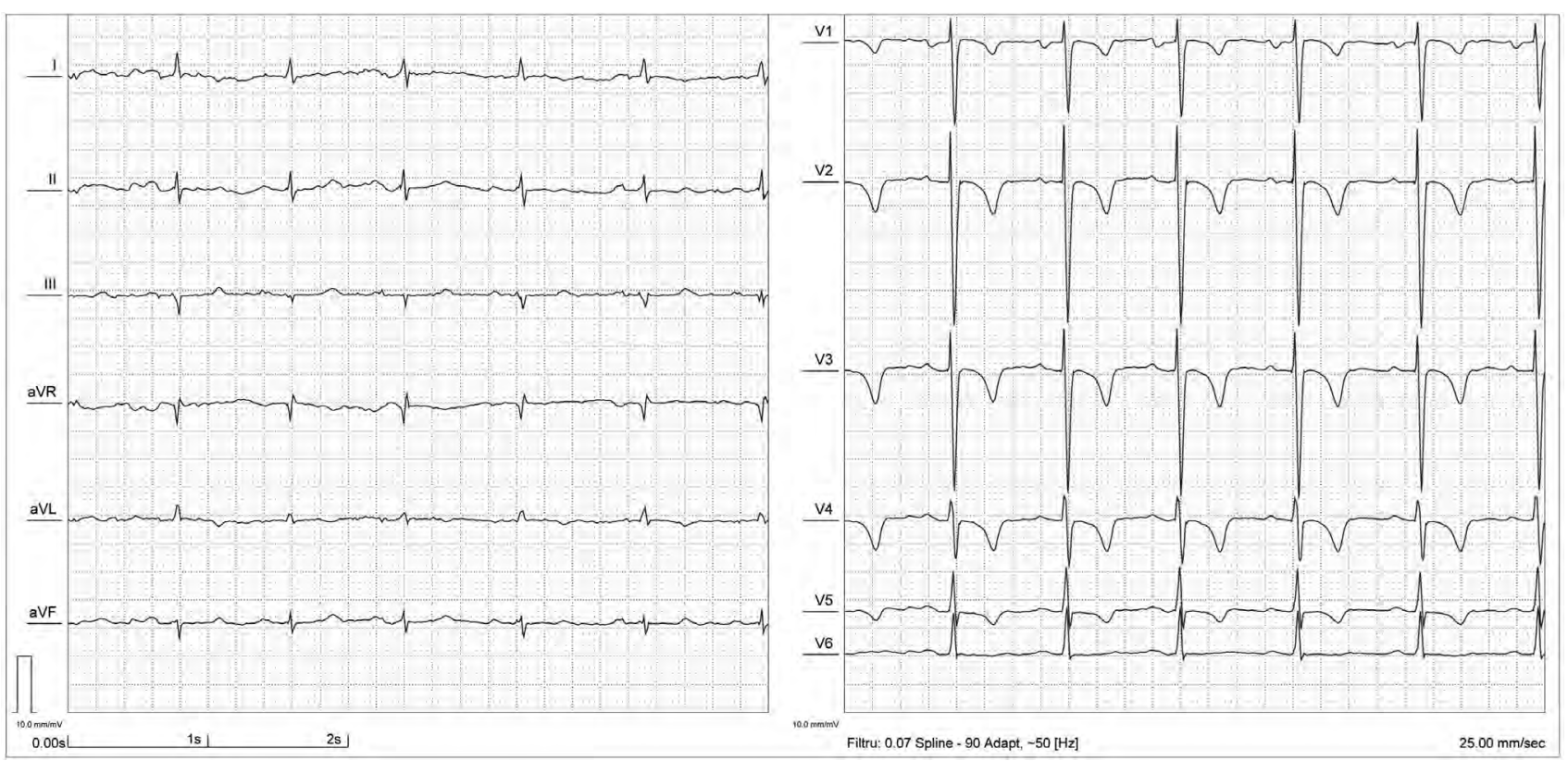

FIGURE 1. 12-lead electrocardiogram (ECG) at first admission (2015)

nary crepitations, a regular cardiac rhythm, mitral holosystolic murmur, and blood pressure of 120/75 $\mathrm{mmHg}$. The 6-minutes walk test highlighted a reduced exercise capacity, a 395 m 6-MWD, from a predicted value of 572 meters.

Routinely laboratory tests were within normal limits except total cholesterol $(6.13 \mathrm{mmol} / \mathrm{l})$, and fibrinogen $(5.9 \mathrm{~g} / \mathrm{l})$. A baseline level of NT-proBNP was also assessed, showing a value of $953.5 \mathrm{pg} / \mathrm{ml}$.

A possible RT-induced subclinical hypothyroidism (TSH: $9.3 \mathrm{mlU} / \mathrm{ml}, \mathrm{FT} 4: 1.09 \mathrm{ng} / \mathrm{dl}$ ) was diagnosed. Serologic patterns suggestive for chronic $B$ or $C$ hepatitis, human immunodeficiency virus, Borrelia burgdorferi, Treponema pallidum, Chlamydia spp. were negative.

Rest electrocardiogram (BTL Industries Ltd, BTL08MT Plus machine, UK) recording identified sinus rhythm with a heart rate of $72 \mathrm{bpm}$, first degree atrio-ventricular block ( $P-Q=204 \mathrm{~ms}$ ), patterns of myocardial ischemia in precordial leads (figure 1). Serial 24-hour Holter ECG recordings revealed rare, isolated supraventricular and ventricular extrabeats.

Chest X-ray indicated a $2.5 \times 3 \mathrm{~cm}$ inhomogeneous, egg-shaped, well-delineated, opacity located above the left pulmonary hilum.

2D transthoracic echocardiography was performed (GE Healthcare, GE Vivid ${ }^{\mathrm{TM}}$ E9 ultrasound system, Boston, MA, USA), according to guidelines recommendations (17). Off-line analysis of stored images showed a dilated left ventricle (LVED: $67 \mathrm{~mm}$ ), moderate/severe mitral regurgitation and a mild secondary pulmonary systolic hypertension (PAPs $=36 \mathrm{mmHg}$ ), intermediate echocardiographic probability parameters of pulmonary hypertension. LV function was assessed, showing reduced LV ejection fraction (LVEF: 30-35\%, modified Simpson's biplane method), and reduced global longitudinal strain (GLS: $-17.2 \%$ ). No retractions, calcifications or fibrotic thickening of valves were noticed. Echocardiographic assessment of pericardium did not confirm pericardial thickening or pericardial effusion.

Cardiac testing with myocardial scintigraphy at rest and during exercise revealed a slightly enlarged LV, a fixed perfusion defect located at the level of the septum, middle and basal segment possible in a shorter interventricular context. Invasive coronary angiography (Allura Xper FD10 X-ray system, Koninklijke Philips N.V, Netherlands) displayed normal epicardial arteries without anatomic variances (figures 2 and 3 ).

Cardiac magnetic resonance (GE Healthcare, CMR, 1.5T Optima ${ }^{T M}$ MR450w MRI scanner, Boston, MA, USA) revealed a hipertrabeculated, slightly dilated left ventricle with an improved LVEF (48\%), the normalized end-diastolic volume of $101 \mathrm{ml} / \mathrm{m}^{2}$, and end-systolic volume of $53 \mathrm{ml} / \mathrm{m}^{2}$, with moderate mitral valve regurgitation (figure 4).

All data suggested cardiotoxicity related to prior anthracycline chemotherapy + RT protocols and a diagnosis of heart failure with reduced ejection fraction (HFrEF) NYHA III functional class, dilated cardiomyopathy, moderate mitral regurgitation, secondary systolic pulmonary hypertension was established. A systematic, rationale, work-up regarding different aetiologies of DCM in this index patient was also considered.

Optimal pharmacological regimen (Carvedilol $3.125 \mathrm{mg}$ b.i.d., Ramipril $5 \mathrm{mg}$ o.d., fixed-dose combination diuretics - Spironolactone $50 \mathrm{mg}+$ Furosemide $20 \mathrm{mg}$ o.d. and selective sinus node If channel inhibi- 


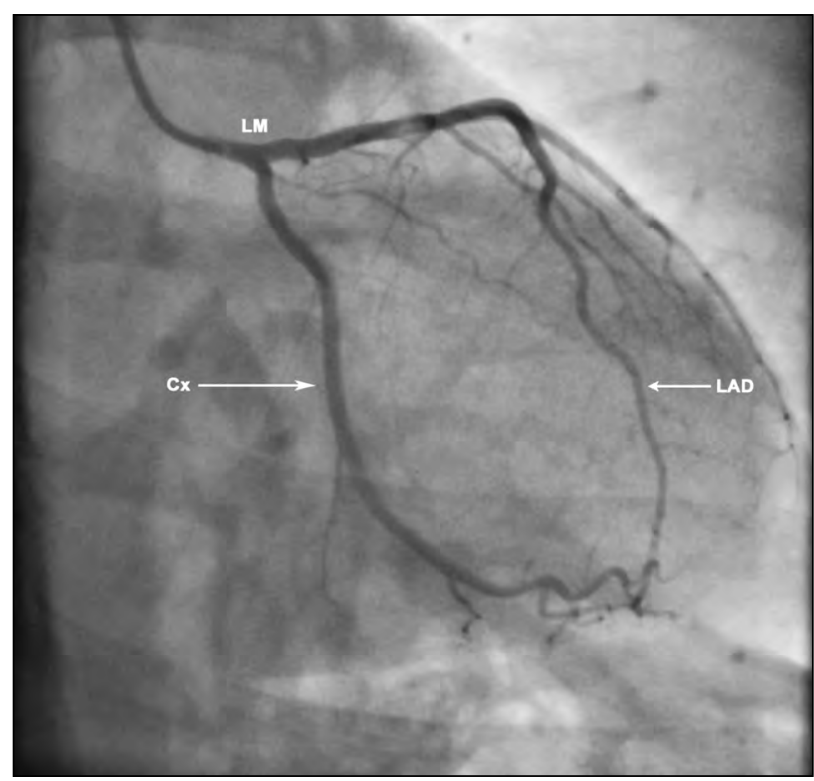

FIGURE 2. Angiographic aspect of left coronary artery ( $L M$ - left main coronary artery, $L A D$ - left anterior descending artery, $C_{X}$ - circumflex artery)

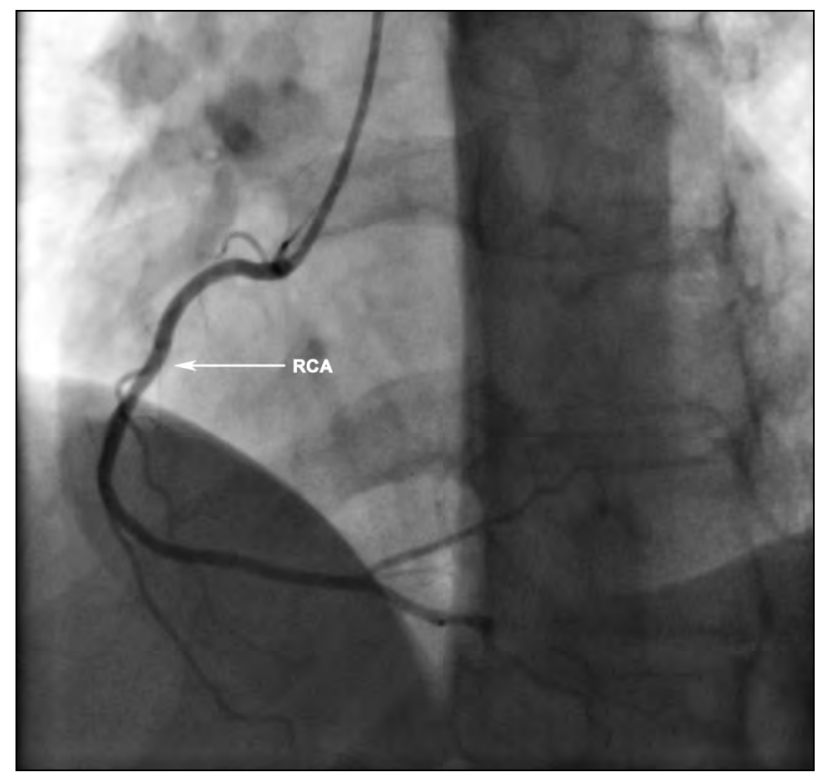

FIGURE 3. Angiographic aspect of right coronary artery (RCA right coronary artery)

tor) was prescribed. Thyroid replacement therapy (Levothyroxine 50 micrograms o.d.) was considered appropriate for subclinical hypothyroidism.

Six months later, a significant improvement of symptoms was noticed, NYHA functional class II, 6-MWD: 530 m (87\% out of predictive 6-MWD of 583 meters), and a $63.27 \%$ NT-proBNP decrease (350.2 pg/ $\mathrm{ml})$.

At four-years regular follow-up (2019), a complete clinical work-up, lab tests and imaging studies (echocardiography and (MR) was performed. Exercise capacity was enhanced (545 meters from a predictive 6-MWD of 567 meters - 96\%) and NT-proBNP dis-

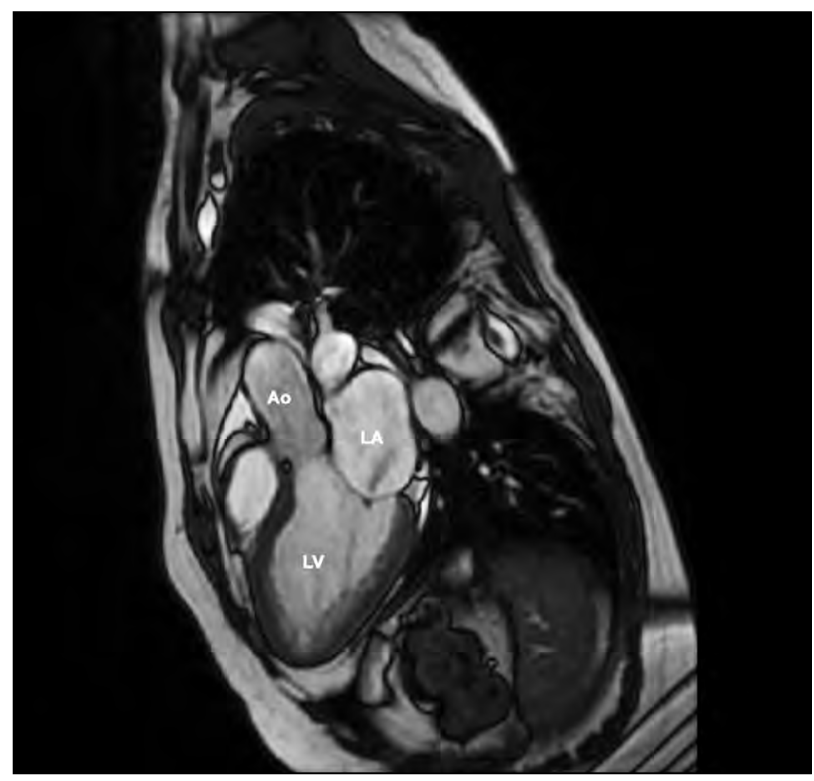

FIGURE 4. Cardiac magnetic resonance imaging at diagnosis (Ao-aorta, LA - left atrium, LV - left ventricle)

played a $237.3 \mathrm{pg} / \mathrm{ml}$ value $(75 \%$ reduction from baseline).

The rest ECG recording showed sinus rhythm, and heart rate of $75 \mathrm{bpm}$, with new-onset of complete left bundle branch block (LBBB) and secondary ST-T changes (figure 5). Serial rest ECG and 24-hours Holter recordings didn't show malignant arrhythmias or Q-T prolongation.

Repeated late gadolinium enhanced CMR revealed DCM, myocardial fibrosis with non-ischemic distribution in myocardial segments $7,8,11,14$ and moderate mitral regurgitation with LVEF of $55 \%$, the indexed end-diastolic volume of $118 \mathrm{ml} / \mathrm{m}^{2}$ and end-systolic volume of $53 \mathrm{ml} / \mathrm{m}^{2}$ respectively (figure 6).

Pharmacological regimen for HF was optimized, increasing the dose of Carvedilol up-to $6.25 \mathrm{mg}$ b.i.d.; statins (Atorvastatin $40 \mathrm{mg}$ o.d.) were added.

No relapse of $\mathrm{HL}$, musculoskeletal disease or other malignancies were identified 24 years after chemotherapy and radiation.

\section{DISCUSSIONS}

Considering the previous medical data, the clinical work-up, lab tests and multimodality imaging, heart failure, dilated cardiomyopathy, mitral regurgitation and intraventricular conduction disturbances reflect late cardiotoxicity given by the chemotherapeutic regimens (MOPP+ABVD) combined with RT (Cobalt-60 mantle therapy). In addition, diffuse myocardial fibrosis without scarring may indicate specific cellular changes anthracycline-induced cardiomyopathy related (18).

Anthracycline-based chemotherapy is the key-element in $\mathrm{HL}$ and other malignancies. However, between 


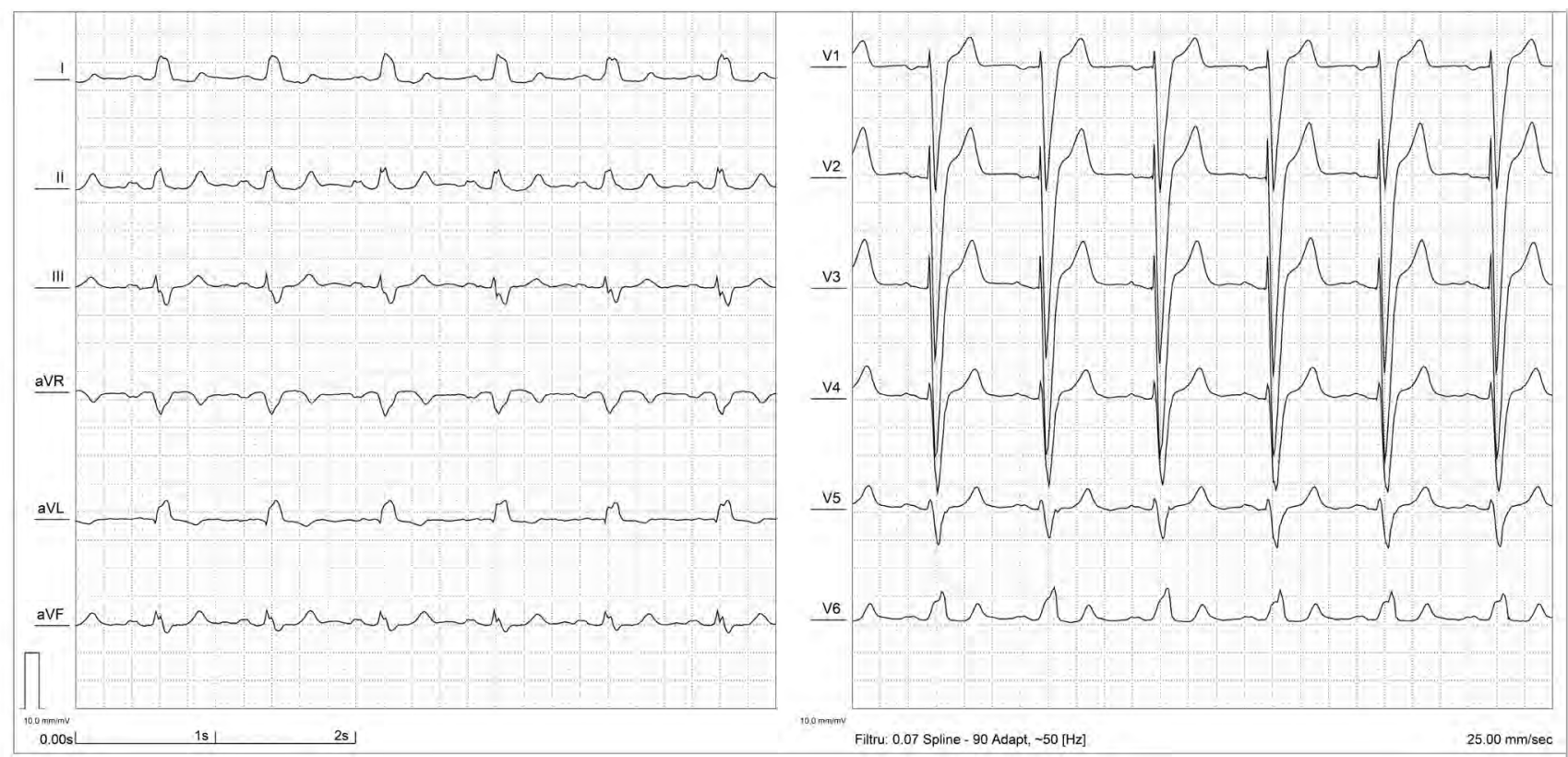

FIGURE 5. 12-lead electrocardiogram (ECG) at 4 years follow-up (2019)

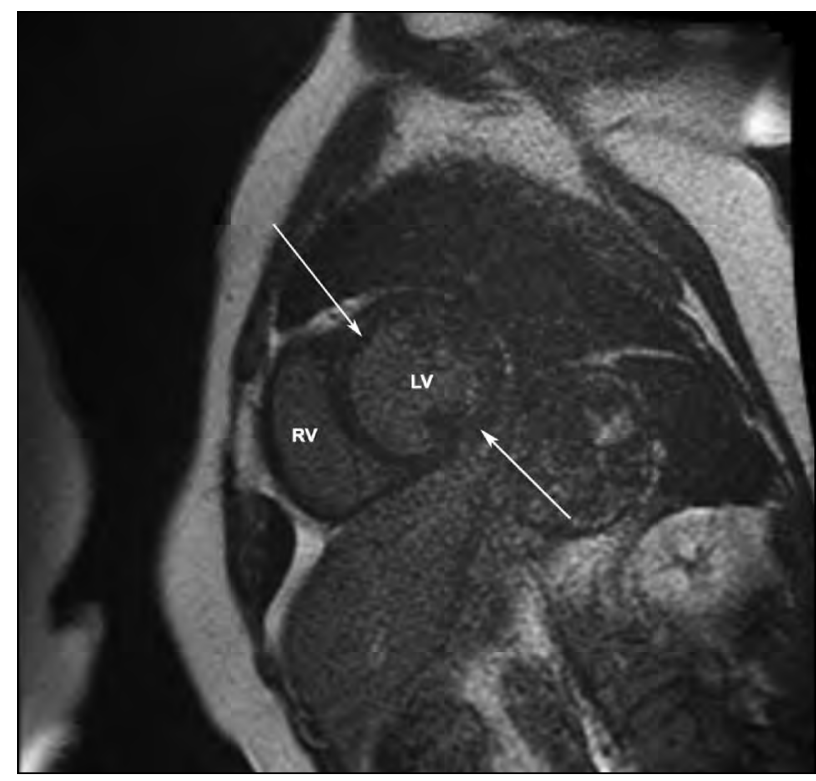

FIGURE 6. Late gadolinium enhanced CMR at 4 years follow-up (2019). Myocardial fibrosis is seen in segments 7,8 11 , and 14 (arrows)

2 and $23 \%$ of patients receiving anthracyclines develop late heart failure, secondary to the induced cardiotoxicity. Despite the normal ejection fraction of the left ventricle, subtle abnormalities of systolic myocardial function are present in long-term survivors after anthracycline exposure.

Anthracyclines and/or RT are involved in the occurrence of mitral regurgitation, a timeframe of more than ten years being attributable to radiotherapy $(9,19-21)$. Hull et al. reported in a retrospective study on $415 \mathrm{HL}$ patients a $6.2 \%$ rate ( 25 cases) of significant valvular dysfunction after a median of 22 years, the aortic valve being most affected (22). The underlying mechanisms of valvular disease secondary to cancer treatments are unclear. Based on imagistic studies performed, we consider that mitral regurgitation is secondary to annular dilation as a consequence of $L V$ dysfunction and remodeling occurred later in the course of CRTCD.

Acute or late-onset of cardiac arrhythmias/conduction system disturbances represents a result of myocardial injury induced by chemo or RT, right bundle branch block is generally more common than LBBB $(23,24)$. In our patient, the first-degree A-V block was present from the first presentation, and after two years follow-up, an asymptomatic left bundle branch block was identified.

Although pericardial disease induced by doxorubicin and radiotherapy is relatively frequent diagnosed $(25,26)$ no features of pericardial involvement were detected by non-invasive imaging.

Treatment including beta-blockers, ACE inhibitors, aldosterone antagonists, statins is the recommended therapeutic regimen, similar to other types of cardiomyopathies with heart failure $(20,27,28)$. Despite data published by Cardinale et al. (29), our patient presented a functional recovery of LV after initiation of personalized HF treatment. Long-term follow-up of cardiac status is desirable, to reduce overtreatment in case of permanently normalized LVEF. Advanced treatment options for HF (cardiac resynchronization therapy/implantable cardioverter-defibrillator) will be considered when advisable. Current evidence do not support the use of anticoagulation in this particular case (no active cancer or evidence of previous thrombotic events, DCM in sinus rhythm), despite the fact that HF confers a prothrombotic state. The hypothesis that FXa inhibi- 
tion can reverse or improve early adverse cardiac remodeling is promising (30); despite the existing specific methods to determine the plasma concentration of direct oral anticoagulants, further research is needed in assumption that these techniques can be used to adjust the drug dosage $(31,32)$.

Pathogenesis of late-onset chronic development cardiotoxicity anthracycline-containing chemotherapy + mediastinal RT related remains unclear, resulting from multifactorial and complex mechanisms, and usually is considered irreversible. Particularly, longterm survivors who experienced $\mathrm{HL}$ in childhood or young age, appear to be susceptible and remain at increased risk of developing heart failure decades after.

Previous therapies contribute to late cardiac involvement or further life-threatening conditions, although the occurrence rate is poorly known, as no precise guidelines are available (33-35).

\section{CONCLUSIONS}

From the setting-up of targeted therapy for Hodgkin lymphoma, cardio-oncology knowledge should be joined, avoiding the late stages, when symptomatic heart failure occurs.

Surveillance of Hodgkin lymphoma patients in complete remission, and personalized care approach are required in order to reduce the constantly increase risk of future cardiovascular events. Patients and doctors should be well-informed about this existing risk; cardioprotective agents are needed, succeeding to lower the enhanced morby-mortality incidence in Hodgkin's lymphoma patients, treated with anthracyclines and radiotherapy.

The authors certify that they have obtained all appropriate patient consent forms. In the forms, the patient has given her signed consent for images and other clinical information to be reported in the journal.

Conflict of interest: none declared Financial support: none declared

\section{REFERENCES}

1. Kang $\mathrm{Y}, \mathrm{Xiao} F$, Chen $\mathrm{H}$ et al. Subclinical Anthracycline-Induced Cardiotoxicity in the Long Term Follow Up of Lymphoma Survivors: A Multi-Layer Speckle Tracking Analysis. Arq Bras Cardiol. 2018; 110:219-28.

2. Valcovici M, Andrica F, Serban C, Dragan S. Cardiotoxicity of anthracycline therapy: current perspectives. Arch Med Sci. 2016; 12:428-35.

3. Virizuela JA, García AM, de Las Peñas R et al. SEOM clinical guidelines on cardiovascular toxicity (2018). Clin Transl Oncol. 2019;21:94-105.

4. Hooning MJ, Botma A, Aleman BM et al. Long-term risk of cardiovascular disease in 10-year survivors of breast cancer. J Natl Cancer Inst. 2007;99:365-75.

5. Boyne DJ, Mickle AT, Brenner DR et al. Long-term risk of cardiovascular mortality in lymphoma survivors: A systematic review and meta-analysis. Cancer Med. 2018; 7:4801-13.

6. Shanbhag S, Ambinder R. Hodgkin Lymphoma: A review and update on recent progress. CA Cancer J Clin. 2018; 68:116-32.

7. Benetou DR, Stergianos E, Geropeppa M et al. Late-onset cardiomyopathy among survivors of childhood lymphoma treated with anthracyclines: A systematic review. Hellenic J Cardiol. 2019;60:152-64.

8. Stone CR, Mickle AT, Boyne DJ et al. Treatment for lymphoma and late cardiovascular disease risk: A systematic review and meta-analysis. Health Sci Rep. 2019;2:135.
9. Plana JC, Galderisi M, Barac A et al. Expert consensus for multimodality imaging evaluation of adult patients during and after cancer therapy: a report from the American Society of Echocardiography and the European Association of Cardiovascular Imaging. Eur Heart J Cardiovasc Imaging. 2014;15:1063-93.

10. Fornaro A, Olivotto I, Rigacci L et al. Comparison of long-term outcome in anthracycline-related versus idiopathic dilated cardiomyopathy: A single centre experience. Eur J Heart Fail. 2018; 20:898-906.

11. Nousiainen $T$, Jantunen $E$, Vanninen $E$, Hartikainen J. Early decline in left ventricular ejection fraction predicts doxorubicin cardiotoxicity in lymphoma patients. Br J Cancer. 2002;86:1697-700

12. Zamorano JL, Lancellotti $P$, Rodriguez Muñoz D et al. 2016 ESC Position Paper on cancer treatments and cardiovascular toxicity developed under the auspices of the ESC Committee for Practice Guidelines: The Task Force for cancer treatments and cardiovascular toxicity of the European Society of Cardiology (ESC). Eur Heart J. 2016;37:2768-801.

13. Henriksen PA. Anthracycline cardiotoxicity: An update on mechanisms, monitoring and prevention. Heart. 2018;104:971-7.

14. van Nimwegen FA, Schaapveld M, Janus $\mathrm{CP}$ et al. Cardiovascular disease after Hodgkin lymphoma treatment: 40-year disease risk. JAMA Intern Med. 2015;175:1007-17.
15. Chatterjee K, Zhang J, Honbo N, Karliner JS. Doxorubicin cardiomyopathy. Cardiology. 2010;115:155-62.

16. Felker GM, Thompson RE, Hare JM et al. Underlying causes and long-term survival in patients with initially unexplained cardiomyopathy. N Engl J Med. 2000; 342:1077-84.

17. Lang RM, Badano LP, Mor-Avi V et al. Recommendations for cardiac chamber quantification by echocardiography in adults: an update from the American Society of Echocardiography and the European Association of Cardiovascular Imaging. Eur Heart J Cardiovasc Imaging. 2015; 16:233-70.

18. Yeh ET, Bickford CL. Cardiovascular complications of cancer therapy: Incidence, pathogenesis, diagnosis, and management. J Am Coll Cardiol. 2009;53:2231-47.

19. Cozzarín A, Cianciulli TF, Saccheri MC et al. Severe mitral regurgitation after radiotherapy. Echocardiography. 2014; 31:E37-40.

20. Donis N, Oury C, Moonen M, Lancellotti P. Treating cardiovascular complications of radiotherapy: A role for new pharmacotherapies. Expert Opin Pharmacother. 2018;19:431-42.

21. Cutter DJ, Schaapveld M, Darby SC et al. Risk of valvular heart disease after treatment for Hodgkin lymphoma. J Natl Cancer Inst. 2015;107.

22. Hull MC, Morris CG, Pepine CJ, Mendenhall NP. Valvular dysfunction and carotid, subclavian, and coronary artery disease in 
survivors of hodgkin lymphoma treated with radiation therapy. JAMA. 2003;290:2831-7.

23. Bovelli D, Plataniotis G, Roila F. ESMO Guidelines Working Group. Cardiotoxicity of chemotherapeutic agents and radiotherapyrelated heart disease: ESMO Clinical Practice Guidelines. Ann Oncol. 2010; 21:277-82.

24. Raghunathan D, Khilji MI, Hassan SA, Yusuf SW. Radiation-Induced Cardiovascular Disease. Curr Atheroscler Rep. 2017;19:22.

25. Casey DJ, Kim AY, Olszewski AJ. Progressive pericardial effusion during chemotherapy for advanced Hodgkin lymphoma. Am. J. Hematol. 2012; 87:521-24.

26. Groarke JD, Nguyen PL, Nohria A et al. Cardiovascular complications of radiation therapy for thoracic malignancies: The role for non-invasive imaging for detection of cardiovascular disease. Eur Heart. J. 2014;35:612-23.
27. Khan N, Husain SA, Husain SI et al. Remission of Chronic Anthracycline-Induced Heart Failure with Support from a Continuous-Flow Left Ventricular Assist Device. Tex Heart Inst J. 2012;39:554-6.

28. Tan TC, Neilan TG, Francis $S$ et al. Anthracycline-Induced Cardiomyopathy in Adults. Compr Physiol. 2015;5:1517-40.

29. Cardinale D, Colombo A, Lamantia G et al. Anthracycline-induced cardiomyopathy: clinical relevance and response to pharmacologic therapy. J Am Coll Cardiol. 2010;55:213-20.

30. Guo X, Kolpakov MA, Hooshdaran B et al. Cardiac Expression of Factor $X$ Mediates Cardiac Hypertrophy and Fibrosis in Pressure Overload. JACC Basic Trans/ Sci. 2020;5:69-83.

31. Varga A, Serban RC, Muntean DL et al. Rapid Liquid Chromatography Tandem Mass Spectrometry Determination of Rivaroxaban Levels in Human Plasma for Therapeutic Drug Monitoring. Rev Romana Med Lab. 2017;25:145-55.
32. Tilea I, Popa DS, Szakács XT et al. Determination of Apixaban Levels in Human Plasma by a High-Throughput Liquid Chromatographic Tandem Mass Spectrometry Assay. Rev Romana Med Lab. 2015;23:115-25.

33. Armenian SH, Hudson MM, Mulder RL et al. International Late Effects of Childhood Cancer Guideline Harmonization Group. Recommendations for cardiomyopathy surveillance for survivors of childhood cancer: A report from the international late effects of childhood cancer guideline harmonization group. Lancet Oncol. 2015;16:123-36.

34. Aleman BM, van den Belt-Dusebout AW, De Bruin ML et al. Late cardiotoxicity after treatment for Hodgkin lymphoma. Blood. 2007;109:1878-86.

35. Lipshultz SE, Adams MJ. Cardiotoxicity after childhood cancer: Beginning with the end in mind. J Clin Oncol. 2010;28:1276-81. 